\title{
MOAGEM SEMI-AUTÓGENA DA USINA DO SOSSEGO - HISTÓRICO DOS 10 ANOS DE OPERAÇÃO E OTIMIZAÇÕES
}

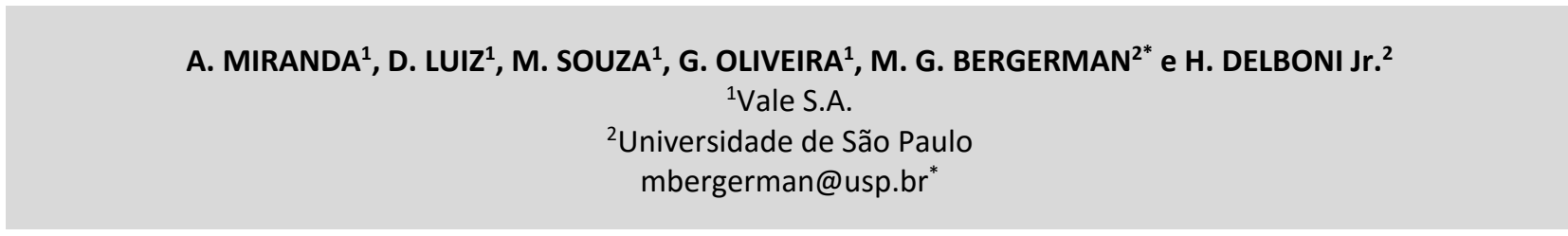 \\ Artigo submetido em novembro/2015 e aceito em novembro/2015 \\ DOI: $10.15628 /$ holos.2015.3656
}

\section{RESUMO}

A usina do Sossego foi a primeira operação da Vale S.A. a operar com um moinho semi-autógeno (SAG) para o processamento de minério sulfetado de cobre e ouro. A usina está localizada no sudeste do estado do Pará. O circuito de cominuição foi projetado para processar 41.000 toneladas por dia de run of mine, sendo o circuito composto por um britador primário, um moinho semiautógeno de 11,6 $\mathrm{m}$ de diâmetro que opera em circuito fechado com britadores cônicos, seguido de dois moinhos de bolas de $6,7 \mathrm{~m}$ de diâmetro. 0 projeto do circuito se baseou em dados de extensa campanha piloto, assim como em simulações baseadas nos resultados de ensaios de caracterização em amostras de furos de sonda. Após o comissionamento do circuito, observou-se que algumas variáveis estavam contribuindo para vazões horárias abaixo das esperadas. Uma série de otimizações e adequações foram realizadas ao longo dos 10 anos de operação do moinho semi-autógeno, incluindo mudança na área aberta, tamanho e disposição das aberturas das grelhas, perfil dos revestimentos e mudanças no plano de fogo da mina. Este trabalho descreve os principais aspectos do projeto do circuito, assim como as otimizações realizadas ao longo dos primeiros anos de operação.

PALAVRAS-CHAVE: Moagem semi-autógena, Simulação, Otimização, Cobre, Geometalurgia.

\section{SOSSEGO SAG MILL - 10 YEARS OF OPERATION AND OPTIMIZATIONS}

\begin{abstract}
Sossego was the first Vale S.A. SAG mill operation to process copper-gold ore. It is located in Para state, south east of Amazon region in Brazil. The comminution circuit was designed to process 41,000 metric tons per day comprising primary crushing, one $38 \mathrm{ft}$ diameter SAG mill operating in closed configuration with cone crushers, followed by two $22 \mathrm{ft}$ diamenter ball mills. The circuit was designed on the basis of data obtained from an extensive pilot plant campaign. The ore variability was also assessed through simulation using data from a drill core
\end{abstract}

characterization program. After commissioning it became clear that a number of variables were contributing to the low throughputs obtained in the SAG mill. Several optimizations and circuit adjustments were made in the 10 initial years of operation, including changes in the grates open area, lifters profile and blasting patterns at the mine. This paper describes the main aspects of designing the circuit, as well as the work carried out at the 10 initial years of operation of the industrial grinding circuit to improve its performance.

KEYWORDS: Semi-autogenous grinding, Simulation, Optimization, Copper, Geometalurgy. 


\section{INTRODUÇÃO}

A mina do Sossego, da Vale S.A., localizada em Canaã dos Carajás, no estado do Pará iniciou suas operações em 2004, sendo a primeira mina de cobre da empresa. Esta possui uma reserva estimada de 245 milhões de toneladas com teores de 0,98\% de cobre e 0,28 g/t de ouro (para um cut off de $0,33 \%$ de cobre equivalente), divididos em dois corpos, denominados Sequeirinho (principal) e Sossego. Este último representa aproximadamente 15\% das reservas. Há também cerca de 16,5 milhões de toneladas de minério de cobre oxidado (VALE S.A., 2001).

O minério de cobre é basicamente calcopíritico, com pequena presença de bornita e calcocita. A usina do Sossego processa 41.000 toneladas de minério de cobre por dia e está projetada para uma produção superior a 470.000 toneladas de concentrado de cobre por ano. 0 fluxograma de processo é apresentado na Figura 1.
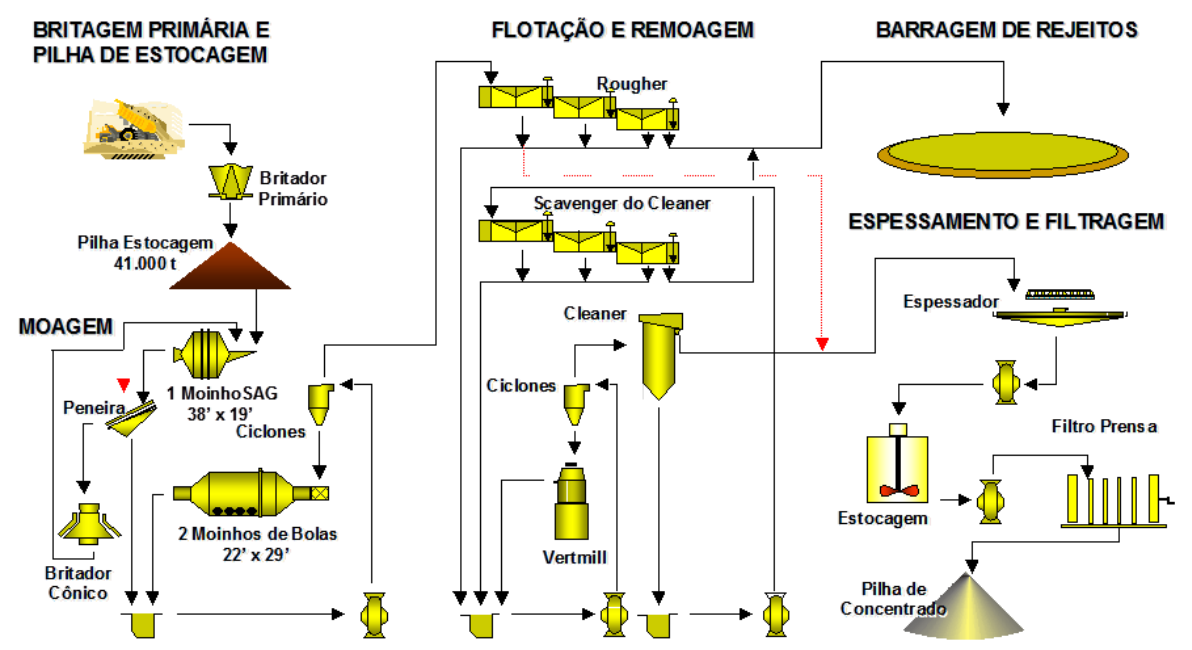

Figura 1. Fluxograma de processo da usina do Sossego (Fonte: ROSA et al.,2007b)

O circuito da usina do Sossego é descrito em detalhes por Bergerman (2009) e Rosa et al. (2007b). Para o dimensionamento do circuito de cominuição, uma extensa campanha de testes em escala piloto foi realizada no Centro de Investigaciones Mineras y Metalurgicas (CIMM) em 2000. Diversas configurações de circuito e condições operacionais foram avaliadas com amostras dos depósitos do Sossego e Sequeirinho. A configuração SABC (moinho SAG em circuito fechado com britador de pebbles) foi selecionada e os equipamentos dimensionados para processor $41.000 \mathrm{t} / \mathrm{d}$. Os resultados da planta piloto deixaram claro que o desempenho do moinho SAG é impactado fortemente pela quantidade de material próximo do tamanho crítico no moinho, confirmando os resultados de características do minério de alta resistência ao impacto, com um valor médio do índice Axb de 32,2 para o minério do Sossego e 49,5 para o minério do Sequeirinho. Adicionalmente aos ensaios em planta piloto, foram conduzidos estudos de variabilidade com amostras de furos de sonda que permitiram a avaliação do comportamento do circuito por meio de simulações com o software JKSimMet. Maiores detalhes sobre o projeto do circuito de cominuição podem ser obtidos em Delboni Júnior et al. (2006).

Após o comissionamento do circuito, observou-se que algumas variáveis estavam contribuindo para vazões horárias abaixo das esperadas. Uma série de otimizações e adequações foram realizadas no moinho semi-autógeno, incluindo mudança na área aberta, tamanho e 
disposição das aberturas das grelhas, perfil dos revestimentos e mudanças no plano de fogo da mina. A seguir são descritas as otimizações mais relevantes realizadas ao longo dos dez primeiros anos de operação.

\section{DESCRIÇÃO DAS OTIMIZAÇÕES REALIZADAS}

\subsection{Modificações nos perfis das grelhas e dos revestimentos}

Uma das principais características observadas ao final de 2004, alguns meses após o início da operação da usina foi a intensa acumulação de material próximo do tamanho crítico, chamado de pebbles, no interior do moinho SAG. Independente da realização de amostragens, visualmente era possível observar uma grande quantidade de material entre 25 e $76 \mathrm{~mm}$ na carga do moinho durante as paradas para inspeção. Na ocasião, a carga circulante era de aproximadamente 20 $25 \%$ da alimentação nova do SAG de aproximadamente $1.100-1.200 \mathrm{t} / \mathrm{h}$. Nesta época, o moinho operava com grelhas de descarga de $8,6 \%$ de área aberta e pebble ports de $63,5 \mathrm{~mm}$. Esta configuração de grelhas operou de março a dezembro de 2004. Desde então, uma série de modificações foram realizadas no projeto das grelhas de forma a aumentar a área aberta das grelhas e a abertura dos pebble ports, de forma a minimizar o acumulo de material próximo do tamanho crítico no interior do moinho. A Tabela 1 ilustra as modificações realizadas no projeto das grelhas até se chegar a configuração considerada ótima.

Tabela 1. Evolução da configuração das grelhas do moinho SAG do Sossego

\begin{tabular}{|c|c|c|c|c|c|c|c|}
\hline Geração & $\begin{array}{l}\text { Pebble port } \\
(\mathrm{mm})\end{array}$ & $\begin{array}{c}\text { Área das } \\
\text { aberturas } \\
\left(\mathrm{cm}^{2}\right)\end{array}$ & Grelhas & $\begin{array}{l}\text { Área total } \\
\left(\mathrm{cm}^{2}\right)\end{array}$ & $\begin{array}{c}\text { Área } \\
\text { aberta** } \\
(\%)\end{array}$ & Período & $\begin{array}{c}\text { Vazão } \\
\text { SAG (t/h) }\end{array}$ \\
\hline Primeira & 63,5 & 2.645 & 32 & 84.627 & 8,6 & Abr - Dez/04 & 1.205 \\
\hline \multirow{2}{*}{$\begin{array}{c}\text { Primeira e } \\
\text { segunda }\end{array}$} & 63,5 & 2.645 & 22 & 58.181 & 8,9 & Dez-fev/05 & 1.247 \\
\hline & 76,2 & 2.974 & 10 & 29.744 & & & \\
\hline Segunda & 76,2 & 2.974 & 32 & 95.181 & 9,7 & Fev-mai/05 & 1.416 \\
\hline \multirow{2}{*}{$\begin{array}{c}\text { Segunda e } \\
\text { terceira }\end{array}$} & 76,2 & 2.974 & 22 & 64.437 & 10,2 & Mai - ago/05 & 1.559 \\
\hline & 88,9 & 3.485 & 10 & 34.852 & & & \\
\hline \multirow{3}{*}{$\begin{array}{c}\text { Primeira, } \\
\text { segunda e } \\
\text { terceira }\end{array}$} & 63,5 & 2.645 & 18 & 47.603 & 9,7 & Ago - set/05 & 1.572 \\
\hline & 76,2 & 2.974 & 1 & 2.974 & & & \\
\hline & 88,9 & 3.485 & 13 & 45.308 & & & \\
\hline \multirow{2}{*}{$\begin{array}{c}\text { Primeira e } \\
\text { terceira }\end{array}$} & 63,5 & 2.645 & 9 & 23.801 & 10,5 & & 1.639 \\
\hline & 88,9 & 3.485 & 23 & 80.160 & & Set-dez/05 & \\
\hline Quarta & 76,2 e 88,9 & 3.352 & 32 & 107.264 & 10,9 & Dez - fev/06 & $*$ \\
\hline Quinta & 76,2 e 88,9 & 3.352 & 32 & 104.264 & 10,9 & Fev $-a b r / 06$ & $*$ \\
\hline Sexta & 76,2 e 88,9 & 3.299 & 32 & 105.568 & 10,7 & Abr-jun/06 & 1.643 \\
\hline Sétima & 76,2 e 88,9 & 3.056 & 32 & 97.792 & 9,9 & Jun - ago/06 & 1.694 \\
\hline
\end{tabular}

A descrição detalhada das modificações realizadas nas grelhas do SAG neste período podem ser encontradas em Delboni Júnior et al. (2006). Estas modificações permitiram subir de um patamar de 1.450 t/h em 2005 para aproximadamente 1.600 t/h em 2006. 
2.2. Otimização da ciclonagem, controle de enchimento do moinho e abertura do circuito de rebritagem do moinho SAG

No ano de 2007, as otimizações no circuito SAG se concentraram na etapa de ciclonagem, no melhor controle operacional do enchimento do moinho e na abertura do circuito do SAG, com a possibilidade de se encaminhar o produto da rebritagem diretamente ao moinho de bolas.

Em relação a ciclonagem um diagnóstico realizado, descrito em detalhes em Bergerman et al. (2011), mostrou que os ciclones estavam trabalhando com o by-pass de finos para o underflow relativamente alto, maiores que $25 \%$. Além disto, amostragens no circuito de flotação mostraram que $25 \%$ do cobre do rejeito da flotação rougher estava liberado e abaixo de $10 \mu \mathrm{m}$. A avaliação do histórico dos dados da recuperação da flotação em relação a granulometria de alimentação mostraram ainda uma melhora de recuperação, com valores de até $96 \%$ de recuperação rougher em granulometrias de alimentação da flotação com aproximadamente $17 \%$ retido em $0,21 \mathrm{~mm}$, um ganho significativo em relação ao patamar observado de $93 \%$ para uma granulometria mais fina, com aproximadamente $12 \%$ retido em $0,21 \mathrm{~mm}$. Em função destas observações, foram realizadas simulações utilizando-se o software JKSimmet, calibrado para o circuito de cominuição do Sossego, que mostram que ganhos poderiam ser obtidos com o uso de um apex menor e um vortex maior, resultando em uma carga circulante menor no circuito e menor geração de finos. Dois ganhos secundários, não previstos inicialmente, foram obtidos. O primeiro se deve ao fato de que com menor carga circulante, a moagem de bolas, que normalmente opera com duas bombas e duas baterias de ciclones, para atingir $100 \%$ da carga nominal, consegue atualmente operar com apenas uma bomba e atingir $95 \%$ da taxa nominal. Antes desta adequação, o valor não passava de $85 \%$. O segundo se deve ao aumento da vida útil dos revestimentos utilizados nos ciclones. Aproveitando a necessidade de mudança nos tamanhos dos revestimentos, foi realizado junto ao fornecedor um teste com novos materiais. Um revestimento cerâmico substituiu o de borracha normalmente utilizado. Apesar de ter um custo 15 vezes maior, sua vida útil é 25 vezes maior, gerando um ganho significativo, ao se levar em conta que existe ainda uma grande economia com mão de obra e minimização das perdas de produção ao se parar menos a bateria para manutenção.

Quanto ao controle de enchimento do moinho SAG, tal demanda surgiu da baixa disponibilidade do mesmo, observada em função de quebras de revestimentos. A avaliação dos dados operacionais mostrou que em alguns momentos o moinho operava com baixa carga, o que facilita o impacto dos corpos moedores, que tem aproximadamente $130 \mathrm{~mm}$, com o revestimento do moinho. Foi estabelecido um controle rígido de enchimento do moinho, que considerava para o cálculo do peso ideal de operação o enchimento de corpos moedores e o desgaste do revestimento, assim como foi implantado um controle da velocidade do moinho, que varia principalmente com o desgaste e perfil do revestimento.

A terceira otimização de destaque neste período foi a abertura do circuito de rebritagem do moinho SAG. A fim de flexibilizar o circuito e melhor equilibrar a energia entre a moagem de bolas e a moagem SAG, uma alternativa operacional foi proposta, criando-se a opção de direcionar o produto dos rebritadores de reciclo (circuito fechado com a moagem SAG) para uma outra etapa de peneiramento. A fração grosseira retornaria para a rebritagem, enquanto a fração fina seria encaminhada diretamente à moagem de bolas. Chamou-se esta alternativa operacional de abertura do circuito da rebritagem de pebbles. 
Os detalhes do projeto e resultados obtidos com a abertura do circuito podem ser obtidos em Rosa et al. (2007a). Estas modificações permitiram um aumento aproximado de $50 \mathrm{t} / \mathrm{h}$ na alimentação do SAG no ano de 2007, com significativo aumento de disponibilidade, em função da significativa redução do número de quebras dos revestimentos.

\subsection{Integração mina usina}

Conforme apontado pela literatura (MORELL e VALERY, 2001), o afinamento da distribuição granulométrica de alimentação de moinho semi-autógeno é um dos pontos que pode gerar maiores ganhos de vazão na moagem, sem necessidade de modificações ou investimentos na usina. A fim de avaliar esta opção, foi realizada uma simulação com as distribuições granulométricas da alimentação da usina dos anos de 2005 e 2006, mais grosseiras do que a observada em uma amostragem de 2007 (Caso Base), dia em que o moinho apresentou uma vazão de alimentação acima da de projeto. As características do minério alimentado foram mantidas como no Caso Base, para evitar a influência desta variável na análise. Não foi realizada nenhuma simulação com alimentação mais fina que a do Caso Base, pois este já estava com uma alimentação considerada fina. A metodologia detalhada para a realização das simulações está descrita em Bergerman (2009). A Tabela 2 ilustra as distribuições granulométricas utilizadas nas simulações, assim como os resultados obtidos.

Tabela 2. Resultados das simulações variando a distribuição granulométrica da alimentação do moinho semiautógeno

\begin{tabular}{ccccc}
\hline Simulação & $\begin{array}{c}\mathrm{F}_{80} \\
(\mathrm{~mm})\end{array}$ & $\begin{array}{c}\text { Vazão de sólidos } \\
\text { de alimentação } \\
(\mathrm{t} / \mathrm{h})\end{array}$ & $\begin{array}{c}\text { Grau de } \\
\text { enchimento } \\
\text { moinho semi- } \\
\text { autógeno (\%) }\end{array}$ & $\begin{array}{c}\text { Potência do } \\
\text { moinho semi- } \\
\text { autógeno (kW) }\end{array}$ \\
\hline $\begin{array}{c}\text { Caso Base - distribuição } \\
\text { granulométrica 2007 }\end{array}$ & 59 & 1.892 & 30,0 & 14.701 \\
\hline $\begin{array}{c}\text { Engrossamento da distribuição } \\
\text { granulométrica de alimentação - } \\
\text { 2006 }\end{array}$ & 74 & 1.100 & 29,9 & 14.457 \\
\hline $\begin{array}{c}\text { Engrossamento da distribuição } \\
\text { granulométrica de alimentação - } \\
\text { 2005 }\end{array}$ & 128 & 700 & 29,9 & 14.285 \\
\hline
\end{tabular}

As simulações realizadas indicam que com o engrossamento da granulometria de alimentação, passando de um $\mathrm{F}_{80}$ de $59 \mathrm{~mm}$ para $128 \mathrm{~mm}$, a vazão de sólidos de alimentação nova do circuito caiu de $1.892 \mathrm{t} / \mathrm{h}$ para 700 t/h, mantendo-se a potência consumida do moinho semiautógeno. Nota-se assim o efeito extremamente significativo e deletério ao desempenho do moinho semi-autógeno e, conseqüentemente, de todo o circuito de moagem do Sossego, do engrossamento da granulometria de alimentação. O patamar observado na amostragem de 2007 pode ser considerado adequado, devendo, portanto, ser mantido, garantindo a vazão nominal de alimentação do circuito, sem a necessidade de investimentos adicionais na usina. A partir das observações realizadas com essa simulação, iniciou-se um trabalho em conjunto com a equipe da mina (BERGERMAN et al., 2008; BERGERMAN, DELBONI e ROSA, 2009), visando à adequação do desmonte na mina, de forma que esta distribuição granulométrica ótima da amostragem de 2007 passasse a ser um valor de referência e de rotina. Foram realizados alguns desmontes em caráter experimental, que confirmaram os resultados ótimos obtidos no dia da amostragem. Desde então, 
com a nova configuração do desmonte, observou-se um aumento médio na vazão da usina no ano de 2008 , em comparação ao ano de 2007 , da ordem de $10 \%$. A mina precisou realizar adequações na malha de desmonte e nas razões de carga utilizadas, assim como na utilização de perfuratrizes. Essas adequações, no entanto, podem ser consideradas de baixo custo frente ao ganho observado na vazão da usina. De forma a se acompanhar a granulometria de alimentação do SAG, foi instalado um analisador on line de granulometria no produto do britador primário, da empresa Split engineering. Com esta adequação, obteve-se uma vazão medida do SAG de $1.731 \mathrm{t} / \mathrm{h}$ no ano de 2007.

\subsection{Troca intercalada de grelhas}

De forma a minimizar o impacto com uma diminuição brusca de área aberta quando se realiza troca das grelhas, foi implementada uma estratégia de troca alternada de grelhas. A Figura 2 ilustra o mapa de troca de grelhas, aonde pode se observar grelhas trocadas em diferentes momentos a cada dois meses.

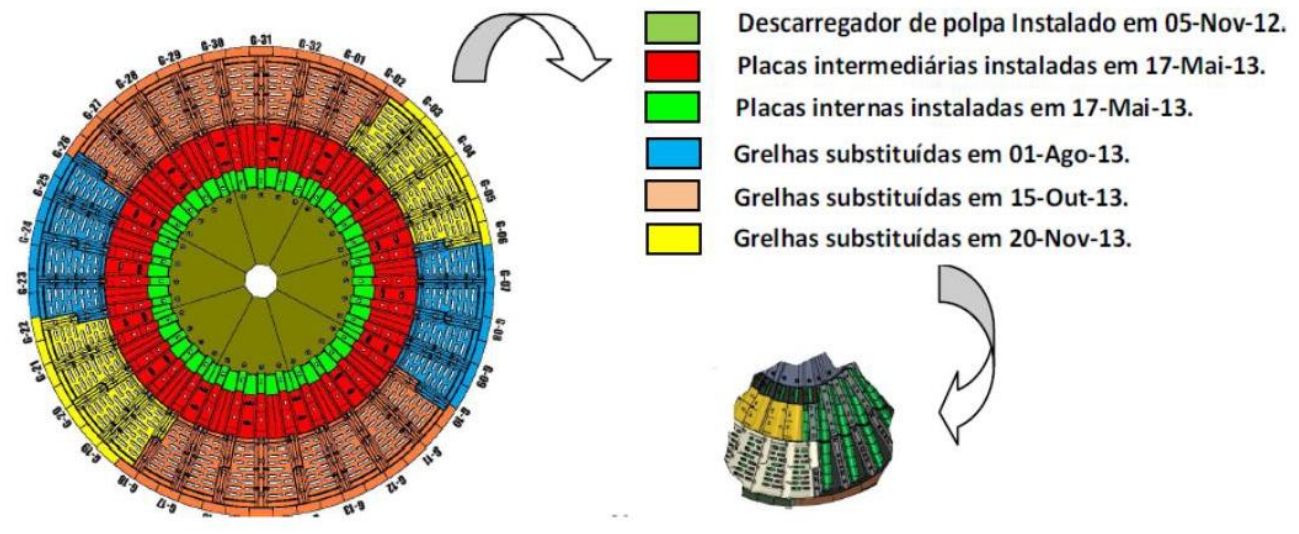

Figura 2. Troca alternada de grelhas do moinho SAG.

Com esta otimização, obteve-se no ano de 2009 uma vazão horária média de 1.766 t/h.

\subsection{Otimizações nos revestimentos e estratégias de operação e manutenção}

Foram implantadas algumas otimizações no revestimento do moinho SAG, como a utilização de grelhas duplas, revestimentos de borracha, a redução do número de placas do cilindro e a reciclagem de placas altas como baixas, além de um rígido controle operacional do enchimento do moinho. A Figura 3 ilustra as modificações nos revestimentos. 


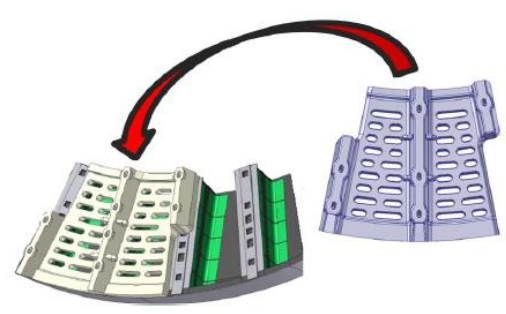

Utilização de grelha dupla e revestimento de borracha



Reciclagem de placas altas antigas como placas baixas


Revestimentos do espelho de alimentação do SAG


Figura 3. Adequações realizadas nos revestimentos do moinho SAG

A Figura 4 ilustra o aumento da massa processada por cada jogo de revestimento do moinho SAG e a diminuição no número de quebras.

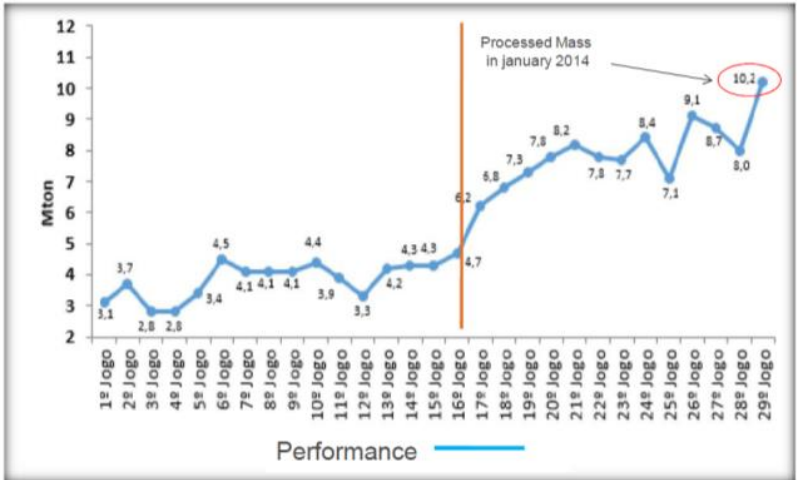

Massa processada por cada jogo de revestimento

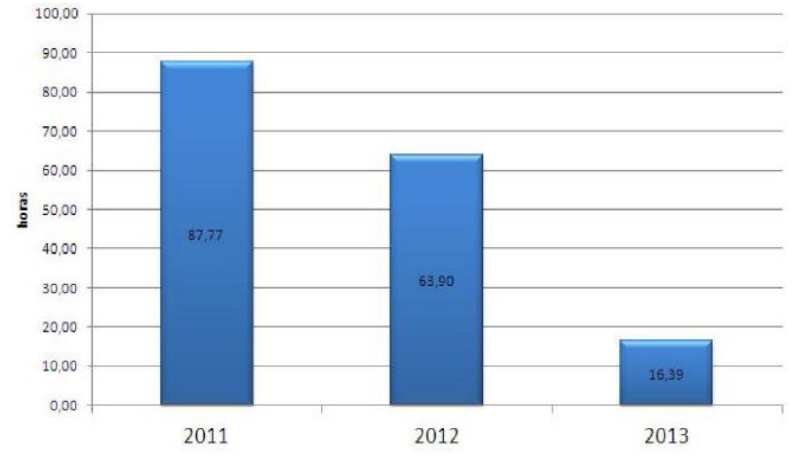

Horas de parada para troca de revestimentos quebrados

Figura 4. Aumento da massa processada por cada jogo de revestimento do moinho SAG e a diminuição no número de quebras

\section{CONCLUSÕES}

O circuito de cominuição do Sossego foi o primeiro do Brasil a operar com um moinho SAG de grande porte. Os primeiros anos de operação apresentaram uma série de desafios a equipe 
técnica envolvida no início da operação e estabilização do circuito. As otimizações implantadas no circuito permitiram que se atingisse de forma consistente a vazão horária projetada para o circuito.

\section{REFERÊNCIAS BIBLIOGRÁFICAS}

1. BERGERMAN, M.G.; MORAIS, J.; CASTRO, A.; ROSA, M.A.N.; DELBONI JR.; H. Variability studies for the Sossego $41 \mathrm{ktpd}$ grinding circuit. In: INTERNATIONAL MINERAL PROCESSING CONFERENCE, 24th, 2008, Beijing. Proceedings. Beijing: Science Press, 2008. v.1, p. 407-416.

2. BERGERMAN, M.G. Modelagem e simulação do circuito de moagem do Sossego. 2009. 207 p. Dissertação (Mestrado) - Escola Politécnica, Universidade de São Paulo, São Paulo, 2009.]

3. BERGERMAN, M.G.; DELBONI, H.; NANKRAN, M. Estudo de variabilidade e otimização do circuito de moagem Sag da usina do Sossego. Revista da Escola de Minas, Ouro Preto, v. 62, n. 1, p. 93-97, jan./mar. 2009.

4. BERGERMAN, M.G.; FONSECA, R.; DELBONI JÚNIOR, H.; DAYRELL, M.; MIRANDA, A., ROSA, M. A. N. Otimização da ciclonagem de 33" do circuito de moagem do Sossego. In: XXIV Encontro Nacional de Tratamento de Minérios e Metalurgia Extrativa. Proceedings do XXIV Encontro Nacional de Tratamento de Minérios e Metalurgia Extrativa; 2011; Salvador, Brasil. p. 895-902.

5. DELBONI JÚNIOR, H.; ROSA, M. A. N.; BERGERMAN, M. G.; NARDI, R. Optimization of the Sossego SAG mill. In: ADVANCES IN AUTOGENOUS AND SEMIAUTOGENOUS GRINDING TECHNOLOGY, 2006, Vancouver. Proceedings.Vancouver: University of British Columbia, 2006. v. 1 , p. I39-150.

6. MORELL, S.; VALERY, W. Influence of feed size on AG/SAG mill performance. In: INTERNATIONAL CONFERENCE ON AUTOGENOUS AND SEMIAUTOGENOUS GRINDING TECHNOLOGY, 2001, Vancouver. Proceedings: SAG 01. Vancouver: University of British Columbia, 2001. v. 1, p. I-203-I-214.

7. ROSA, M. A. N.; BERGERMAN, M. G.; FONSECA, R. A.; QUEIROZ, A.; GERALDO, J. Abertura do circuito de moagem da usina do sossego, In: XXII Encontro Nacional de Tratamento de Minérios e Metalurgia Extrativa. Proceedings do XXII Encontro Nacional de Tratamento de Minérios e Metalurgia Extrativa; 2007; Ouro Preto, Brasil. v. I. p. 123-126. (a)

8. ROSA, M.A.N., BERGERMAN, M.G., MIRANDA, A., OLIVEIRA, J.L., SOUZA, M., BATISTA FILHO, J., CARDOSO, W. Controle operacional da usina do Sossego. In: Meeting of the southern hemisphere on mineral technology e Encontro nacional de tratamento de minérios e metalurgia extrativa. Proceedings do VII Meeting of the southern hemisphere on mineral technology e XXII Encontro nacional de tratamento de minérios e metalurgia extrativa. Ouro Preto: Universidade Federal de Ouro Preto, 2007. v. III. p. 505-512. (b)

9. VALE S.A.. Relatório de viabilidade do Projeto Sossego. Carajás: Minerconsult, Bechtel, ECM e Concremat, 2001. 451 p. (Relatório interno). 